\title{
Intervenciones nutricionales en mujeres lactantes infectadas por el VIH
}

\author{
Saurabh Mehta ${ }^{a, b}$ Julia L. Finkelstein ${ }^{a}$ Wafaie W. Fawzi ${ }^{a, b}$

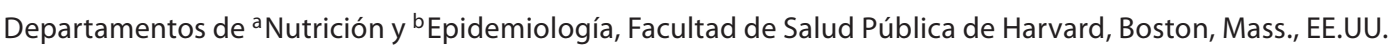

\section{Palabras clave}

Nutrición $\cdot \mathrm{VIH} \cdot \mathrm{SIDA} \cdot$ Multivitaminas $\cdot$ Amamantamiento

\section{Extracto}

El amamantamiento es el patrón de oro internacional de la alimentación del lactante, pues es sabido que reduce el riesgo de morbilidad y mortalidad en menores de un año y fomenta mejoras del crecimiento y el desarrollo de los niños. No obstante, en el contexto del VIH, la lactancia materna incrementa significativamente el riesgo de transmisión de madre a hijo/a (TMAH) del $\mathrm{VIH}$, riesgo que es proporcional a la duración del amamantamiento. En consecuencia, la Organización Mundial de la Salud recomienda evitar el amamantamiento por parte de mujeres infectadas por el VIH cuando la alimentación sustitutiva es aceptable, factible, asequible, sostenible y segura. Desafortunadamente, la adopción de la alimentación con leches para lactantes no es profusa en regiones en vías de desarrollo, como África subsahariana, debido en parte a su elevado coste y en parte al estigma social asociado al hecho de no amamantar. Además, en entornos donde el acceso a condiciones higiénicas adecuadas es limitado, la alimentación con leches para lactantes puede dar lugar a una mayor morbilidad y mortalidad en los menores de un año. En consecuencia, el amamantamiento sigue siendo responsable de una proporción considerable de infecciones pediátricas por VIH en regiones en vías de desarrollo del mundo. Algunos estudios basados en la observación, destinados a examinar el papel del estado nutricional en la transmisión vertical del VIH, hallaron que niveles séricos bajos de vita- mina A se asociaban a un mayor riesgo de TMAH. No obstante, en dos ensayos realizados en Malawi y Sudáfrica, respectivamente, la vitamina A careció de efecto significativo sobre la TMAH, mientras que en ensayos realizados en Tanzania y Zimbabwe se comprobó que el aporte complementario de vitamina $A$ a las madres aumentaba significativamente el riesgo de TMAH del VIH. En Tanzania, el aporte complementario de multivitaminas (vitaminas B, C y E) a las madres reducía el riesgo de transmisión del VIH a través del amamantamiento en mujeres afectadas nutricional e inmunológicamente y producía beneficios adicionales sobre la salud, como una menor incidencia de pérdidas fetales y una reducción de la tasa de progresión de la enfermedad materna. En conjunto, no se dispone de datos para respaldar el aporte complementario de vitamina A en madres gestantes infectadas por el VIH. No obstante, el aporte complementario de multivitaminas, incluyendo las vitaminas $B, C$ y $E$, se recomienda fehacientemente. Está garantizada la realización de investigación adicional para indagar el papel que desempeñan otros nutrientes en la transmisión vertical del VIH a través del amamantamiento y en la salud global materna y del lactante en mujeres lactantes infectadas por el VIH y su descendencia. En entornos donde no son viables las alternativas a la lactancia materna, se recomienda el amamantamiento exclusivo durante seis meses en niños nacidos de madres infectadas por el VIH, conjuntamente con un destete rápido y una alimentación sustitutiva óptima para la salud del lactante.

Copyright $\odot 2007$ Nestec Ltd., Vevey/S. Karger AG, Basel

\section{KARGER}

Fax +4161306 1234 E-Mail karger@karger.ch www.karger.com (c) 2007 Nestec Ltd., Vevey/S. Karger AG, Basel 0252-8185/07/0651-0039\$23.50/0

Accesible online en: www.karger.com/ans
Wafaie W. Fawzi, MD, DrPH

Department of Nutrition, Building II Room 329A

Harvard School of Public Health, 651 Huntington Avenue

Boston, MA 02115 (USA)

Tel. +1 617432 5299, Fax +1 617432 2435, E-Mail mina@hsph.harvard.edu 


\section{Introducción}

Se estima que en todo el mundo 1,800 niños llegan a infectarse diariamente por el VIH, con aproximadamente 650,000 nuevas infecciones pediátricas y 500,000 muertes relacionadas con el SIDA cada año $[1,2]$. Sobre el África subsahariana recae la mayor parte de la carga global de la patología pediátrica por VIH/SIDA, que comprende más del $85 \%$ de las nuevas infecciones por VIH en niños en todo el mundo. En 2003, el SIDA fue responsable del $6.5 \%$ de las muertes en niños menores de 5 años en África, lo que representa un incremento a partir del $2 \%$ registrado en 1990 [2]. La mayoría de las infecciones pediátricas por VIH se adquieren a través de la transmisión de madre a hijo/a (TMAH), bien sea en el útero, durante el parto o durante el amamantamiento.

En los países desarrollados, estrategias como el tratamiento antirretroviral (TAR) y la alimentación exclusiva con leches para lactantes han reducido satisfactoriamente las tasas de la transmisión vertical del VIH hasta por debajo del 2\% [3]. No obstante, en ausencia de TAR e intervenciones nutricionales en regiones en vías de desarrollo, los niños presentan un riesgo del 25 al 48\% de contraer una infección por VIH a partir de sus madres infectadas por este virus [4]. De hecho, el amamantamiento sigue siendo responsable de 300,000 nuevas infecciones pediátricas cada año en países en vías de desarrollo, o aproximadamente un $40 \%$ de todas las TMAH del VIH [5].

En consecuencia, la Organización Mundial de la Salud (OMS) recomienda evitar el amamantamiento por parte de madres infectadas por el VIH 'cuando la alimentación sustitutiva es aceptable, factible, asequible, sostenible y segura' [6]. No obstante, la alimentación sustitutiva exclusiva es en general inviable en zonas menos desarrolladas, debido a la falta de agua potable, a condiciones higiénicas insuficientes, al elevado coste de las leches para lactantes, al estigma social y al mayor riesgo de morbilidad y mortalidad en los menores de un año [7-9]. Además, las regiones en vías de desarrollo se enfrentan a una precariedad de servicios de asistencia prenatales, incluyendo el asesoramiento voluntario y las pruebas de VIH, así como el acceso al TAR. Por ejemplo, aunque se estima que la sero-prevalencia del VIH oscila entre el 15 y el 30\% en servicios prenatales de numerosos países de África subsahariana, se estima que la cobertura asistencial media de la prevención de la TMAH es inferior al 6\% [2]. En este contexto, las intervenciones sanitarias preventivas, incluyendo la alimentación del lactante y el asesoramiento nutricional, así como la provisión de TAR, llegan a ser cada vez más importantes para reducir la TMAH del VIH y fomentar mejoras de la salud materna e infantil.

En este artículo examinamos el papel que desempeña la nutrición en mujeres lactantes infectadas por el VIH y su descendencia. Comenzamos con una breve revisión de los beneficios del amamantamiento y las necesidades nutricionales globales durante la lactancia. La relación entre nutrición, VIH y amamantamiento se explora detalladamente revisando los datos disponibles de estudios basados en la observación y ensayos aleatorizados. Por último, concluimos con una discusión de las implicaciones de los hallazgos en la practica clínica y de salud pública.

\section{Amamantamiento por parte de mujeres no infectadas por el VIH}

\section{Beneficios del amamantamiento}

El amamantamiento está acreditado como el patrón de oro internacional de la alimentación del lactante debido a sus beneficios, tanto para la madre como para el lactante. Entre los beneficios para la madre destacan el espaciamiento entre sus hijos, el bienestar psicológico, una recuperación más oportuna del peso anterior al embarazo, una reducción del riesgo de hemorragia posparto y menores tasas de cáncer de mama y cáncer ovárico premenopáusicos [10].

El amamantamiento confiere beneficios nutricionales e inmunológicos al lactante, que comprenden la reducción de la morbilidad y la mortalidad causadas por diarrea e infecciones respiratorias agudas [11]. Por ejemplo, en los países en vías de desarrollo los bebés alimentados con leches para lactantes experimentan un aumento de la mortalidad de 3 a 10 veces superior, causado por diarrea y neumonía, en comparación con los lactantes que reciben exclusivamente leche materna $[12,13]$. De hecho, según una reciente revisión, sólo los programas de promoción del amamantamiento podrían prevenir directamente los 1.3 millones de muertes infantiles estimadas al año en todo el mundo [14]. Se ha demostrado que este mayor riesgo de morbilidad y mortalidad debido a enfermedades infecciosas asociadas a la alimentación con leches para lactantes persiste incluso en el contexto de un acceso modesto al agua potable y a la higiene [15]. Una posible explicación de esta observación es que la alimentación con leches para lactantes puede privar al niño de los beneficios inmunológicos protectores del amamantamiento, incluyendo el aporte de citocinas y anticuerpos [16-18]. El amamantamiento también confiere beneficios al crecimiento y desarrollo del lactante, incluyendo la intensificación de las funciones neurocognitivas en neonatos prematuros con muy bajo peso al nacer [19], atribuible probablemente al contenido en ácidos grasos poliinsaturados de la leche materna [20]. Además, el amamantamiento reduce el riesgo de otitis media e infecciones de las vías urinarias en el lactante [10, $13,21]$.

\section{Necesidades nutricionales durante el amamantamiento}

Con el propósito de apoyar al metabolismo materno y al crecimiento y desarrollo del lactante, las necesidades nutricionales de la madre aumentan durante la lactancia en comparación con el periodo prenatal. Por ejemplo, durante sus 4 a 6 primeros meses de vida, los lactantes doblan el peso que tenían
40

Ann Nestlé [Esp] 2007;65:39-48
Mehta/Finkelstein/Fawzi 
al nacer [22]. Las necesidades maternas de macronutrientes son también superiores durante la lactancia y son directamente proporcionales a la intensidad y la duración del amamantamiento. Las normas actuales recomiendan una ingesta adicional de $500 \mathrm{kcal} /$ día durante los 6 meses iniciales de lactancia y $400 \mathrm{kcal} /$ día en lo sucesivo [22, 23].

Durante el periodo de lactancia también aumentan las necesidades de micronutrientes de la madre y del lactante. En el contexto de una ingestión calórica insuficiente, la ingesta materna de calcio, magnesio, cinc, folato y vitaminas $B_{6}, B_{12}$ y D puede reducirse [24]. En particular, puede ser necesario un aporte complementario de vitaminas $\mathrm{B}_{12} \mathrm{y} \mathrm{D}$ a la madre durante el periodo de lactancia, especialmente cuando la alimentación materna no incluye productos de origen animal $[22,24]$. Hasta la fecha, la investigación se ha centrado fundamentalmente en el papel de las vitaminas $\mathrm{B}_{12} \mathrm{y}$ folato debido a su asociación a enfermedades carenciales congénitas manifiestas. No obstante, los efectos de las carencias de micronutrientes sobre la salud materna y del lactante durante el periodo de lactancia han sido pasados por alto durante mucho tiempo.

\section{Amamantamiento por parte de mujeres infectadas por el VIH}

\section{TMAH del VIH a través del amamantamiento}

Al principio se adujo la transmisión vertical del VIH a través de la leche materna cuando los lactantes adquirían la infección por VIH de mujeres que experimentaban una seroconversión tras el parto por exposición sexual o transfusión sanguínea $[25,26]$. Esta aserción era respaldada por informes subsiguientes de transmisión del VIH a lactantes a través del procedimiento de amamantamiento por nodrizas o por el uso de mezclas de leche materna ajena sin otra fuente conocida de exposición al VIH [27].

Tal como se ha mencionado anteriormente, intervenciones como el TAR y la alimentación exclusiva con leches para lactantes han reducido las tasas de la TMAH del VIH hasta menos del $2 \%$ en países desarrollados como Estados Unidos [3]. No obstante, en los países en vías de desarrollo, en los que el amamantamiento y el VIH son muy prevalentes, la contribución proporcional de la TMAH a la carga de la enfermedad por VIH es considerablemente superior.

El riesgo de TMAH del VIH a través del amamantamiento está influido por factores maternos y del lactante. Por ejemplo, características maternas como los niveles elevados del ARN del VIH en el plasma y la leche materna, el deterioro del estado inmunitario (por ejemplo, recuentos bajos de células CD4), la enfermedad clínica avanzada y el estado nutricional deficiente incrementan la probabilidad de transmisión vertical del VIH [28-30]. Las características víricas pueden afectar también el riesgo de TMAH del VIH. Por ejemplo, en un estudio de parejas madre-lactante infectados por VIH, únicamente se trans- mitieron a los lactantes amamantados los virus que no eran inductores de sincitio y presentaban un mayor tropismo por los macrófagos [31, 32]. La presencia de procesos inflamatorios, como la mastitis materna y los abscesos mamarios, también contribuyen al riesgo de exposición de los lactantes al virus y la transmisión subsiguiente del VIH $[33,34]$. Factores del lactante, como candidiasis y úlceras orales $[33,34]$ y la integridad epitelial del tracto gastrointestinal [35], también influyen sobre el riesgo de exposición al VIH y el riesgo de transmisión $[33,34]$.

Los procedimientos de alimentación de los lactantes también modulan el riesgo de TMAH del VIH. En varios estudios se ha demostrado que la tasa de transmisión del VIH aumenta acumuladamente con la duración del amamantamiento durante todo el periodo postnatal $[36,37]$. Es probable que esta observación sea atribuible al incremento de la exposición de los lactantes al virus. Por ejemplo, en el estudio multicéntrico, Estudio Internacional de Amamantamiento y Transmisión del $\mathrm{VIH}$, realizado en lactantes VIH negativos a las 4 semanas de edad, la tasa de transmisión del VIH a través del amamantamiento fue del $0.74 \%$ por mes, o un riesgo del $4 \%$ durante los 6 meses de duración del amamantamiento [5].

La cronología de la seroconversión materna puede afectar también al riesgo de transmisión vertical del VIH. Por ejemplo, en un estudio prospectivo de cohortes en Ruanda, los investigadores observaron una tasa de transmisión del VIH del $80 \%$ en lactantes nacidos de mujeres que experimentaron seroconversión en el curso de 3 meses tras el parto, en comparación con una tasa de transmisión del $40 \%$ en lactantes cuyas madres llegaron a infectarse entre 4 y 21 meses después del parto [38]. No obstante, se ha descrito que la transmisión del VIH a través del amamantamiento ocurre incluso en un momento tan tardío como 36 meses después del parto [39].

La modalidad de alimentación del lactante influye también sobre la probabilidad de TMAH del VIH; por ejemplo, en varios estudios, el amamantamiento mixto ha mostrado el riesgo máximo de transmisión del VIH en comparación con el amamantamiento a corto plazo exclusivo y la alimentación con leches para lactantes [40-42]. Downs y Cooper [43] han revisado pormenorizadamente estos estudios, junto a otros que relacionan factores maternos y del lactante con el riesgo de transmisión vertical del VIH a través de la lactancia.

\section{Efectos del amamantamiento sobre el estado nutricional y la mortalidad en mujeres infectadas por el VIH}

Se han realizado varios estudios basados en la observación y ensayos aleatorizados para examinar los efectos del amamantamiento sobre la salud materna en mujeres infectadas por el VIH. Por ejemplo, en un estudio basado en la observación realizado en Sudáfrica se comparó el estado nutricional y la composición corporal entre 44 mujeres lactantes VIH negativas y 17 VIH positivas. Entre las mujeres lactantes VIH negativas y VIH positivas no se registraron diferencias significati- 
vas en los parámetros siguientes: peso, índice de masa corporal, circunferencia en los tercios medio y superior del brazo y espesor del pliegue cutáneo en el tríceps [44].

En un ensayo clínico aleatorizado, realizado en Kenia, se asignaron aleatoriamente 425 mujeres infectadas por el VIH al grupo de amamantamiento o al grupo de alimentación con sucedáneos. Los investigadores compararon el peso materno en situación basal (es decir, dos semanas a tres meses después del parto) y en el seguimiento (es decir, de cinco a nueve meses después del parto) y evaluaron la mortalidad durante todo el periodo de seguimiento. Las madres lactantes perdieron en promedio $0.17 \mathrm{~kg} / \mathrm{mes}$, mientras que las mujeres del grupo alimentando con sucedáneos no perdieron peso $(\mathrm{p}=0.03)$ [45]. Se registró también un incremento tres veces superior del riesgo de mortalidad en las mujeres lactantes en comparación con las mujeres del grupo de alimentación con sucedáneos. Los investigadores postularon que el incremento de la mortalidad observado en el grupo de lactancia materna probablemente era debido a una depleción de los nutrientes maternos. Sin embargo, los bajos niveles de cumplimiento pueden haber atenuado la asociación observada entre el estado alimentario del lactante y el riesgo subsiguiente de mortalidad materna, lo que limita la fiabilidad interpretativa de los datos. Por ejemplo, el $4 \%$ de las mujeres asignadas al grupo de lactancia materna no proporcionaron nada de leche materna a sus bebés, y el $29 \%$ de las mujeres asignadas al grupo de alimentación con sucedáneos amamantaron también a sus bebés. Se registraron también diferencias significativas en las características basales entre los grupos de lactancia materna y alimentación con leches para lactantes, incluyendo una carga vírica mediana superior en el plasma de las mujeres asignadas aleatoriamente al amamantamiento, lo cual sería de esperar que incrementase el riesgo de TMAH del VIH en el grupo de lactancia materna. Además, la tasa de transmisión intrauterina del VIH fue mayor en el grupo de lactancia materna, lo que puede ser atribuible al grado avanzado de la enfermedad en las mujeres lactantes. No obstante, los autores no proporcionaron información alguna sobre la progresión de la enfermedad clínica en ambos grupos [46]. Además, no se conocen mecanismos biológicos plausibles que expliquen un efecto del amamantamiento sobre la mortalidad materna [47].

La asociación entre amamantamiento y el riesgo de mortalidad no ha podido reproducirse en otros estudios de campo en mujeres lactantes VIH positivas. Por ejemplo, en un estudio en 566 mujeres infectadas por el VIH, realizado en Sudáfrica, el estado de lactancia materna no influyó sobre el riesgo de mortalidad materna; fallecieron 2 de 410 mujeres $(0.49 \%)$ que siempre habían amamantado, en comparación con 3 de 156 (1.92\%) que nunca lo habían hecho [47]. Análogamente, en una cohorte de tanzanas, el estado o la duración de la lactancia materna no guardaba relación con el riesgo de mortalidad materna, pérdida de peso, anemia o cambios en los recuentos de células CD4 [48]. Además, no se observó ninguna asociación entre el estado de lactancia materna y el riesgo de mortalidad materna en estudios realizados en Zambia [49], Malawi [50] o en un estudio multicéntrico realizado por el Grupo de Estudio Internacional sobre Amamantamiento y Transmisión del VIH [51].

\section{Papel de la nutrición en mujeres lactantes infectadas por el $\mathrm{VIH}$}

Se espera que los beneficios de la mejora de la nutrición materna sean dobles. En primer lugar, puede reducir la TMAH del VIH a través del amamantamiento, bien sea como intervención coadyuvante en mujeres que ya estén recibiendo TAR o como estrategia autónoma para mujeres que se encuentren en una etapa preTAR de la enfermedad. En segundo lugar, es probable que las intervenciones nutricionales mejoren la salud materna y la calidad de vida, así como el crecimiento y el desarrollo de los lactantes. Por ejemplo, en ensayos controlados realizados en hombres y mujeres infectados por el VIH en Canadá [52], Tailandia [53] y Tanzania [54], así como en varios estudios basados en la observación y bien diseñados [55], se demostró el beneficio del aporte complementario de micronutrientes sobre la progresión clínica de la enfermedad causada por el VIH. En consecuencia, las intervenciones nutricionales, como el aporte complementario de micronutrientes, pueden actuar como un coadyuvante útil del TAR o constituir estrategias autónomas en mujeres lactantes infectadas por el VIH para fomentar mejorías de la salud materna e infantil.

Hay varios mecanismos potenciales por medio de los cuales el aporte complementario de nutrientes a la madre puede conferir beneficios tanto a la madre como al lactante, entre los que destacan la mejoría de la salud y la reducción del riesgo de transmisión vertical del VIH. Por ejemplo, los micronutrientes son importantes en el fomento de la integridad epitelial del tejido mamario materno, que afecta la exposición del lactante al virus y al riesgo de transmisión del VIH. En particular, en varios estudios se ha demostrado que la carencia de vitamina A en células epiteliales mamarias resulta en colonias de células de forma irregular con polaridad anormal o en proteínas de la unión intercelular [56-58]. Análogamente, el aporte complementario de micronutrientes puede fortalecer la integridad de la mucosa del tracto gastrointestinal del lactante y afectar la función de las células T y B maternas y del lactante [59-61]; estos efectos pueden reducir subsiguientemente la vulnerabilidad a la infección causada por el VIH. Por ejemplo, en un estudio realizado en Sudáfrica en niños nacidos de madres infectadas por el VIH, la presencia de respuestas de los linfocitos $\mathrm{T}$ cooperadores a las proteínas de cubierta del VIH en la sangre del cordón umbilical produjo efectos protectores frente a la transmisión del VIH a través de vías intraparto y de lactancia materna [62]. La leche materna humana también confiere beneficios nutricionales e inmunológicos al lactante, incluyendo factores que pueden ser protectores frente a la infección por VIH, como inmunoglobulinas, lactoferrina, lisozima, oligosacáridos y glucosaminoglicanos. Los beneficios 
de estos factores inmunológicos en la leche materna pueden incrementarse adicionalmente mejorando el estado nutricional materno a través del aporte complementario de micronutrientes $[59,63]$.

\section{Efecto del estado nutricional materno sobre la TMAH del VIH a través del amamantamiento}

\section{Estudios basados en la observación}

La investigación disponible sobre el papel que desempeña la nutrición como determinante de los desenlaces de salud en mujeres lactantes VIH positivas y su descendencia es escasa. En estudios basados en la observación se ha examinado predominantemente el papel de los micronutrientes en la TMAH del VIH global, con enfoque sobre la vitamina A. Para los propósitos de este artículo revisamos los estudios basados en observación que investigaron la TMAH del VIH global, que incluye las vías intraútero, la exposición intraparto y de lactancia materna. Aunque estos estudios no fueron diseñados para examinar específicamente la transmisión del VIH a través del amamantamiento, se evaluaron resultados seleccionados al cabo de seis semanas del parto, lo que incluiría cierta contribución de la transmisión del VIH a través de la lactancia materna.

En Kenia se realizó un estudio basado en la observación con objeto de evaluar la relación entre el estado materno de vitamina A y el riesgo de transmisión vertical del VIH. Los investigadores examinaron un total de 212 muestras de leche materna procedentes de 107 mujeres lactantes infectadas por el VIH. En la muestra conjunta no se registró asociación alguna entre el estado materno de vitamina A y la presencia del ADN del VIH en la leche materna; no obstante, niveles séricos bajos de vitamina $A(<20 \mu \mathrm{g} / \mathrm{dl})$ se asociaban a un incremento 20 veces superior del riesgo de detectar el ADN del VIH en la leche materna de una submuestra de mujeres inmunodeficientes (es decir, recuentos de células $\mathrm{CD} 4<400 / \mathrm{mm}^{3}$ ); índice de probabilidad = 19.7; intervalo de confianza del 95\% [IC 95\%]: 2.1; 188.5) [64].

Se realizó un estudio en Malawi para examinar la relación entre el estado materno de vitamina A y la transmisión vertical del VIH. En 338 mujeres gestantes infectadas por el VIH, la tasa acumulada de TMAH del VIH fue del $21.9 \%$ al cabo de 12 meses del parto. Las tasas observadas de transmisión vertical del VIH fueron máximas en las mujeres que presentaban los niveles séricos mínimos de vitamina A [65].

La asociación observada entre el estado materno de vitamina A y el riesgo de transmisión vertical del VIH puede ser atribuible a factores alternativos de confusión. Por ejemplo, una infección puede contribuir a la reducción de la movilización de los depósitos hepáticos de vitamina $\mathrm{A}$ y, en consecuencia, a la reducción de sus niveles séricos $[66,67]$. Por lo tanto, los niveles bajos de vitamina A pueden constituir un marcador de la etapa avanzada de la enfermedad causada por el VIH y explicar el incremento observado del riesgo de transmisión del VIH.
Puede existir también un elemento engañoso residual debido a factores adicionales no controlados en estos estudios basados en la observación, incluyendo otras deficiencias nutricionales, infecciones oportunistas y un acceso limitado a la asistencia sanitaria. Otra limitación de estos estudios basados en la observación es la falta de indagación de la vía o la cronología de la transmisión vertical del VIH, que restringe la interpretación de datos referentes a la contribución específica de la lactancia materna a la TMAH del VIH.

\section{Estudios del aporte complementario}

Los resultados y las limitaciones de los estudios basados en la observación mencionados anteriormente alentaron a la realización de ensayos aleatorizados y controlados para investigar a mayor profundidad la relación entre el aporte complementario nutricional y los desenlaces de la salud materna y del lactante en mujeres VIH positivas.

En un ensayo realizado en Sudáfrica se asignó aleatoriamente a 728 mujeres gestantes infectadas por el VIH al aporte complementario diario de vitamina A o placebo durante todo el periodo prenatal. El aporte complementario de vitamina A consistió en la administración diaria de 5,000 UI de vitamina A (1.67 mg de equivalente de retinol) y $30 \mathrm{mg}$ de $\beta$ caroteno (5 mg de equivalente de retinol) durante todo el tercer trimestre de embarazo, y 200,000 UI de vitamina A administradas en el momento del parto. No se registraron diferencias en el riesgo de infección por VIH, ni en la tasa de mortalidad fetal o del lactante entre ambos grupos, al cabo de tres meses después del parto [68]. En una submuestra de 551 mujeres gestantes infectadas por el VIH, que fueron seguidas durante 15 meses después del parto o hasta el cese de la lactancia materna, el aporte complementario prenatal de vitamina A no redujo el riesgo de transmisión del VIH a través de la lactancia materna [42]. En un análisis de los niveles del ARN del VIH en muestras de leche materna en un subgrupo de 79 mujeres infectadas por el VIH, se encontró que la carga vírica se asociaba rotundamente al riesgo de TMAH del VIH. No obstante, el aporte complementario de vitamina A a la madre no redujo significativamente el riesgo de transmisión vertical del VIH [69].

En un ensayo realizado en Malawi se asignó aleatoriamente a 697 mujeres gestantes infectadas por el VIH a la administración diaria de hierro y folato durante todo el embarazo hasta el momento del parto, bien sea solos o en combinación con vitamina A (3 mg de retinol). Todas las mujeres recibieron también $30 \mathrm{mg}$ de retinol al cabo de 6 semanas después del parto como parte del protocolo asistencial. No se registraron diferencias entre ambos grupos en las tasas globales de TMAH del VIH al cabo de 6 semanas del parto (vitamina A: 26.6\%; placebo: $27.8 \%$; $\mathrm{p}=0.76$ ) ni al cabo de 24 meses del parto (vitamina A: 27.7\%; placebo: $32.8 \%$; $\mathrm{p}=0.21$ ). No obstante, el aporte complementario de vitamina $\mathrm{A}$ a la madre se asociaba a una reducción de la tasa de TMAH del VIH en un subgrupo de lactantes que fueron VIH negativos por la reacción de cadenas de polimerasas (RCP) al cabo de 6 semanas. Este efecto 
observado era presumiblemente atribuible a una reducción de la transmisión del VIH a través del amamantamiento $(2.8$ frente a $7.7 \%$ al cabo de 24 meses; $\mathrm{p}=0.04$ ). No obstante, en este ensayo no se examinó específicamente el estado de lactancia materna, lo cual restringe la interpretación de los resultados [70]. Además, los autores hallaron que el aporte complementario de vitamina A a la madre durante el embarazo no influía significativamente sobre los niveles de carga del virus $\mathrm{VIH}$ en la leche materna en un subgrupo de estas mujeres [33].

En el ensayo ZVITAMBO, realizado en Zimbabue, se asignó aleatoriamente a madres infectadas por VIH y sus bebés a la administración diaria de un aporte complementario único de dosis elevadas de vitamina A y/o placebo en el curso de las $96 \mathrm{~h}$ del parto, según un diseño factorial 2 por 2. Los cuatro brazos de la intervención fueron los siguientes: vitamina A tanto a la madre como al lactante; vitamina A a la madre y placebo al lactante; placebo a la madre y vitamina A al lactante; placebo tanto a la madre como al lactante. Un total de 2,060 lactantes VIH negativos fueron seguidos durante todo el periodo posparto con el objeto de evaluar la tasa de transmisión postnatal del VIH (es decir, seroconversión en lactantes que fueron VIH negativos al cabo de seis semanas del parto). Durante el periodo de seguimiento, un $12.1 \%$ de los lactantes experimentaron seroconversión; el aporte de una dosis alta de vitamina A única no redujo el riesgo de transmisión postnatal del VIH [71]. La vitamina A tampoco produjo efectos sobre la mortalidad en los lactantes que fueron RCP positivos para el VIH en la situación basal. En los lactantes que fueron RCP negativos en situación basal, pero RCP positivos al cabo de seis semanas, el aporte complementario neonatal de vitamina A redujo la mortalidad de los lactantes en un $28 \%(\mathrm{p}=0.01)$, si bien el aporte complementario a la madre no produjo efectos sobre la mortalidad de esos lactantes. Por el contrario, en los lactantes que fueron RCP negativos al cabo de seis semanas, el aporte complementario de vitamina A a la madre y al lactante produjo un incremento significativo, dos veces superior, de la mortalidad ( $\mathrm{p} \leq 0.05$ ).

En el estudio Ensayo de Vitaminas (TOV) realizado en Dar es Salaam, Tanzania, 1,078 mujeres gestantes infectadas por el VIH fueron asignadas aleatoriamente a las 12 a 27 semanas gestacionales para recibir la administración diaria, en un diseño factorial 2 por 2 , de vitamina A, multivitaminas, combinación de vitamina A y multivitaminas o placebo. El aporte complementario de vitamina A consistió en $30 \mathrm{mg}$ de $\beta$-caroteno ( $5 \mathrm{mg}$ de equivalente de retinol) y 5,000 UI de vitamina A preformada (1.667 $\mathrm{mg}$ de equivalente de retinol); el aporte complementario de multivitaminas incluía complejo vitamínico $\mathrm{B}$, vitamina $\mathrm{C}$ y vitamina $\mathrm{E}$ (20 $\mathrm{mg}$ de tiamina, $20 \mathrm{mg}$ de riboflavina, $25 \mathrm{mg}$ de vitamina $\mathrm{B}_{6}, 100 \mathrm{mg}$ de niacina, $50 \mu \mathrm{g}$ de vitamina $B_{12}, 500 \mathrm{mg}$ de vitamina $C, 30 \mathrm{mg}$ de vitamina $\mathrm{E}$ y $0.8 \mathrm{mg}$ de ácido fólico). Las mujeres de los grupos de aporte complementario de vitamina A recibieron también una dosis oral de vitamina A $(200,000 \mathrm{UI})$ en el momento del parto, mientras que las mujeres de los grupos de multivitaminas y placebo recibieron un aporte complementario de placebo. Como parte del protocolo asistencial, todas las mujeres recibieron también comprimidos de $120 \mathrm{mg}$ de hierro ferroso y tabletas de $5 \mathrm{mg}$ de folato al día, así como $300 \mathrm{mg}$ de cloroquina como profilaxis del paludismo semanalmente. Ni el aporte complementario de multivitaminas (riesgo relativo $[\mathrm{RR}]=$ 0.95; IC 95\%: 0.73 ; 1.24) ni el aporte complementario de vitamina A $(\mathrm{RR}=1.06$; IC 95\%: 0.81; 1.39) influyeron sobre el riesgo de transmisión del VIH ni sobre la supervivencia de los lactantes hasta seis semanas después del parto. El aporte complementario de multivitaminas a las madres, pero no el de vitamina A, incrementó el peso al nacer de los lactantes [72] y resultó en reducciones significativas, del $40 \%$ aproximadamente, de los riesgos de muerte fetal, bajo peso al nacer $(<2,500 \mathrm{~g})$, premadurez grave $(<34$ semanas gestacionales) y tamaño reducido para la edad gestacional [73]. Las multivitaminas, pero no la vitamina $\mathrm{A}$, resultaron también en un incremento significativo de los recuentos maternos de las células CD4, CD8 y CD3 [73].

En el ensayo TOV, el aporte complementario de vitamina A a las madres produjo un incremento significativo del $38 \%$ del riesgo de transmisión vertical global del VIH, mientras que las multivitaminas carecieron de un efecto similar [74]. El aporte complementario de multivitaminas redujo también significativamente la transmisión del VIH a través de la lactancia materna y mejoró la supervivencia exenta de VIH en niños nacidos de mujeres afectadas nutricional o inmunológicamente (es decir, cuartil mínimo del recuento total de linfocitos) en situación basal, y permanecieron VIH negativos al cabo de seis semanas.

En el estudio TOV, los investigadores examinaron también diversos marcadores clínicos e inmunológicos de progresión de la enfermedad en mujeres infectadas por el VIH durante todo el periodo de seguimiento posparto. El aporte complementario de multivitaminas redujo el riesgo de progresión al estadio IV de la OMS y de la mortalidad relacionada con el SIDA en aproximadamente un $30 \%(\mathrm{RR}=0.71$; IC 95\%: 0.51; 0.98) [54]. Las multivitaminas también redujeron significativamente la incidencia de manifestaciones orales y gastrointestinales maternas de la enfermedad causada por el VIH e instancias de fatiga, erupciones e infecciones agudas de las vías respiratorias superiores. Los aportes complementarios de multivitaminas aumentaron significativamente los recuentos maternos de las células CD4 y CD8, redujeron el nivel de carga vírica [54] y mostraron un efecto protector frente al desgaste en mujeres infectadas por el VIH [75]. El aporte complementario exclusivo de vitamina A no produjo mejorías similares de estos desenlaces [73].

Los efectos adversos observados en el brazo de vitamina $\mathrm{A}$ en el ensayo en Tanzania pueden ser atribuibles al componente $\beta$-caroteno del régimen. Aunque se ha comprobado que el $\beta$-caroteno puede ser relativamente seguro a corto plazo en individuos infectados por el VIH, la seguridad del aporte complementario a largo plazo no ha sido investigada [76, 77]. Ciertos conocimientos recientes de la seguridad a largo plazo, así 
como de la eficacia terapéutica, de los carotenos proceden de un ensayo de intervención en pacientes adultos con SIDA avanzado realizado en Canadá, el Grupo de Estudio de Carotenoides CTN 091/CRIT [78]. Los investigadores de este estudio asignaron aleatoriamente a 166 pacientes del grupo de control a un aporte complementario estándar de micronutrientes (incluyendo vitamina A y oligoelementos) y a 163 pacientes del grupo de tratamiento al aporte complementario estándar de micronutrientes más carotenoides mixtos (equivalente a $72 \mathrm{mg}$ de $\beta$-caroteno), diariamente durante 18 meses. No se observaron efectos adversos de los carotenoides; de hecho, se halló un incremento estadísticamente no significativo de la mortalidad en los participantes que no recibieron carotenoides en comparación con los tratados con estas sustancias. Este incremento llegó a ser significativo en la corrección para los recuentos basales de CD4 y las concentraciones séricas de caroteno (índice de riesgo del tiempo hasta la muerte $=3.15$; IC 95\%: 1.10; 8.98). No obstante, una de las limitaciones principales de este estudio fue la potencia notablemente reducida de la dosis del aporte complementario administrado; los autores comunicaron que la dosis diaria de la medicación en estudio era equivalente a sólo $9.2 \mathrm{mg}$ de $\beta$-caroteno durante el curso del estudio en lugar de los $72 \mathrm{mg}$ propuestos.

Los efectos adversos de la vitamina A observados sobre la transmisión vertical del VIH en los ensayos de Tanzania y Zimbabwe pueden deberse también al efecto de la vitamina $\mathrm{A}$ sobre el genoma del VIH; la vitamina A podría modular potencialmente la replicación del VIH a través de un elemento de respuesta al ácido retinóico en el genoma vírico [79]. La vitamina A puede también causar una mayor densidad de los receptores CCR5 al incrementar la multiplicación y la diferenciación de las células linfoides y mieloides; estos receptores CCR5 son cruciales para la fijación del virus a los linfocitos y la replicación vírica subsiguiente $[71,80]$. Además, la vitamina A puede incrementar el riesgo de transmisión del VIH acelerando la tasa de progresión de la enfermedad causada por el VIH en la madre. Por ejemplo, en un extenso estudio de cohortes basado en la observación, realizado en Estados Unidos, los investigadores observaron una relación en forma de U entre la ingestión alimentaria y complementaria de vitamina $\mathrm{A}$ y el riesgo de progresión al SIDA clínico y la muerte [81, 82]. Concretamente, los cuartiles mínimo y máximo de la ingestión de vitamina $A$ se asociaban a un incremento significativo de la progresión al SIDA en comparación con los dos cuartiles medianos.

Existen dos notables diferencias entre el estudio TOV de Tanzania y los demás estudios mencionados anteriormente. En primer lugar, en el ensayo de Tanzania el aporte complementario a la madre siguió más allá del periodo prenatal, lo que incrementa la importancia de los resultados para las mujeres lactantes infectadas por VIH y sus bebés. En segundo lugar, los investigadores examinaron los efectos de los aportes complementarios de micronutrientes sobre las vías específicas de transmisión vertical, incluyendo la intrauterina, intraparto y las modalidades de lactancia materna. Los resultados demos-

Intervenciones nutricionales en mujeres lactantes infectadas por el VIH traron los efectos beneficiosos del aporte complementario de multivitaminas sobre tres aspectos: el estado nutricional e inmunitario de la madre; la TMAH del VIH a través del amamantamiento y los desenlaces de crecimiento y desarrollo, morbilidad y mortalidad de los lactantes.

\section{Conclusiones}

El amamantamiento confiere beneficios tanto a la madre como al lactante, entre los que destacan la mejoría de la salud materna e infantil y la mejora del crecimiento y desarrollo del lactante. No obstante, en el contexto del VIH/SIDA, la lactancia materna incrementa también el riesgo de TMAH del VIH en forma proporcional a la duración del amamantamiento. Hay factores adicionales que exacerban el riesgo de transmisión vertical del VIH, entre los que conviene mencionar la modalidad y la pauta de alimentación del lactante, el estado nutricional e inmunológico de la madre y el estadio de la enfermedad causada por el VIH en la madre.

Las normas de la OMS recomiendan prescindir del amamantamiento por parte de madres infectadas por el VIH cuando la alimentación sustitutiva es aceptable, factible, asequible, sostenible y segura. En los países en vías de desarrollo, donde no se dan estas condiciones, la OMS recomienda el amamantamiento exclusivo durante los seis primeros meses seguido de un cese brusco.

Se dispone de muy escasa investigación sobre el papel que desempeña la nutrición en mujeres lactantes infectadas por el VIH y su descendencia. Los datos actuales no respaldan el aporte complementario de vitamina A en mujeres gestantes o lactantes infectadas por el VIH, basándose en el incremento de la transmisión vertical del VIH observado en los ensayos de Tanzania y Zimbabwe. No obstante, el aporte diario de multivitaminas a la madre, con la inclusión de las vitaminas B, C y E, se recomienda fehacientemente durante la duración completa de los periodos prenatal y posparto con el objeto de reducir la transmisión vertical del VIH, retrasar la progresión de la enfermedad por VIH en la madre y mejorar la salud materna e infantil. Es probable que este procedimiento sea particularmente beneficioso en mujeres que se encuentran en las fases iniciales de la enfermedad por VIH y que no son todavía elegibles para el TAR; todavía estamos pendientes de averiguar la eficacia y la seguridad del aporte complementario de multivitaminas en individuos que ya estén recibiendo el TAR.

\section{Lagunas en la investigación y orientaciones futuras}

Está justificada la realización de investigación ulterior para examinar el papel que desempeña la nutrición en la salud materna e infantil en mujeres lactantes VIH positivas y su descendencia. Concretamente, es imprescindible investigar adicio- 
nalmente el mecanismo preciso de la transmisión del VIH a través de la lactancia materna; esto contribuiría a identificar los factores nutricionales y de otro tipo que pueden ser los más importantes para reducir la TMAH del VIH. También es necesario determinar el impacto que ejercen factores nutricionales sobre los procesos que aumentan el riesgo de transmisión del VIH, como la mastitis.

En consecuencia, se precisa proceder a una investigación adicional para determinar el aporte complementario de micronutrientes apropiado para mujeres lactantes infectadas por el VIH y sus bebés. Concretamente, es imprescindible aclarar la interacción entre el TAR y los factores nutricionales incluyendo el aporte complementario de micronutrientes.
También está justificado investigar la elaboración de planes de alimentación alternativos viables para los lactantes. Esto llega a ser especialmente importante a las luces de los datos recientes que demuestran la contribución de la alimentación complementaria o sustitutiva inadecuada al incremento del riesgo de retraso o impedimento del crecimiento en lactantes nacidos de mujeres infectadas por el VIH [83]. La transmisión a las madres de asesoramiento nutricional y de alimentación de los lactantes es, en consecuencia, muy prioritario para asegurar el cese precoz del amamantamiento y la instauración subsiguiente de procedimientos de alimentación adecuados para mujeres infectadas por el VIH que optan por amamantar.

\section{Bibliografía}

1 UNAIDS: AIDS Epidemic Update: 2004. Geneva, UNAIDS, 2004.

2 UNAIDS: AIDS Epidemic Update: 2005. Geneva, UNAIDS, 2005.

3 Mofenson LM: Successes and challenges in the perinatal HIV-1 epidemic in the United States as illustrated by the HIV-1 serosurvey of childbearing women. Arch Pediatr Adolesc Med 2004;158:422-425.

4 Dabis F, Msellati P, Dunn D, et al: Estimating the rate of mother-to-child transmission of HIV. Report of a workshop on methodological issues Ghent (Belgium), 17-20 February 1992. The Working Group on Mother-toChild Transmission of HIV. AIDS 1993;7: 1139-1148.

5 Coutsoudis A, Dabis F, Fawzi W, et al: Late postnatal transmission of HIV-1 in breastfed children: an individual patient data meta-analysis. J Infect Dis 2004;189:21542166.

6 WHO: New Data on the Prevention of Mother-to-Child Transmission of HIV and Their Policy Implications: Conclusions and Recommendations. Geneva, UNFPA/WHO/ UNAIDS Inter-Agency Task Team on Mother-to-Child Transmission of HIV, 2001.

$\checkmark 7$ Coutsoudis A, Pillay K, Spooner E, et al: Morbidity in children born to women infected with human immunodeficiency virus in South Africa: does mode of feeding matter? Acta Paediatr 2003;92:890-895.

8 Shapiro RL, Lockman S, Thior I, et al: Low adherence to recommended infant feeding strategies among HIV-infected women: results from the pilot phase of a randomized trial to prevent mother-to-child transmission in Botswana. AIDS Educ Prev 2003;15: 221-230.

9 Eide M, Myhre M, Lindbaek M, Sundby J, Arimi P, Thior I: Social consequences of HIV-positive women's participation in prevention of mother-to-child transmission programmes. Patient Educ Couns 2006;60: 146-151.
10 Dermer A: Breastfeeding and women's health. J Womens Health 1998;7:427-433.

11 Kuhn L, Stein Z, Susser M: Preventing mother-to-child HIV transmission in the new millennium: the challenge of breast feeding. Paediatr Perinat Epidemiol 2004;18:10-16.

12 Nicoll A, Newell ML, Van Praag E, et al: Infant feeding policy and practice in the presence of HIV-1 infection. AIDS 1995;9:107119.

13 WHO: Effect of breastfeeding on infant and child mortality due to infectious diseases in less developed countries: a pooled analysis. WHO Collaborative Study Team on the Role of Breastfeeding on the Prevention of Infant Mortality. Lancet 2000;355:451-455.

14 Jones G, Steketee RW, Black RE, et al: How many child deaths can we prevent this year? Lancet 2003;362:65-71.

15 Habicht JP, DaVanzo J, Butz WP: Mother's milk and sewage: their interactive effects on infant mortality. Pediatrics 1988;81:456461.

16 Garofalo RP, Goldman AS: Cytokines, chemokines, and colony-stimulating factors in human milk: the 1997 update. Biol Neonate 1998;74:134-142.

17 Goldman AS: The immune system of human milk: antimicrobial, antiinflammatory and immunomodulating properties. Pediatr Infect Dis J 1993;12:664-671.

18 Xanthou M, Bines J, Walker WA: Human milk and intestinal host defense in newborns: an update. Adv Pediatr 1995;42:171208.

19 Gordon N: Nutrition and cognitive function. Brain Dev 1997;19:165-170.

20 Weinberg GA: The dilemma of postnatal mother-to-child transmission of HIV: to breastfeed or not? Birth 2000;27:199-205.
21 Villalpando S, Hamosh M: Early and late effects of breast-feeding: does breast-feeding really matter? Biol Neonate 1998;74:177191.

22 Picciano MF: Pregnancy and lactation: physiological adjustments, nutritional requirements and the role of dietary supplements. J Nutr 2003;133:1997S-2002S.

23 Wilson PR, Pugh LC: Promoting nutrition in breastfeeding women. J Obstet Gynecol Neonatal Nurs 2005;34:120-124.

24 Jensen RG: Handbook of Milk Composition. San Diego, Academic Press, 1995

25 Ogundele MO, Coulter JB: HIV transmission through breastfeeding: problems and prevention. Ann Trop Paediatr 2003;23:91106.

26 Palasanthiran P, Ziegler JB, Stewart GJ, et al: Breast-feeding during primary maternal human immunodeficiency virus infection and risk of transmission from mother to infant. J Infect Dis 1993;167:441-444.

27 Nduati RW, John GC, Kreiss J: Postnatal transmission of HIV-1 through pooled breast milk. Lancet 1994;344:1432.

28 Temmerman M, Nyong'o AO, Bwayo J, et al: Risk factors for mother-to-child transmission of human immunodeficiency virus-1 infection. Am J Obstet Gynecol 1995;172: 700-705.

29 Thorne C, Newell ML: Prevention of mother-to-child transmission of HIV infection. Curr Opin Infect Dis 2004;17:247-252.

30 Mayaux MJ, Blanche S, Rouzioux C, et al: Maternal factors associated with perinatal HIV-1 transmission: the French Cohort Study: 7 years of follow-up observation. The French Pediatric HIV Infection Study Group. J Acquir Immune Defic Syndr Hum Retrovirol 1995;8:188-194.

- 31 Ometto L, Zanotto C, Maccabruni A, et al: Viral phenotype and host-cell susceptibility to HIV-1 infection as risk factors for motherto-child HIV-1 transmission. AIDS 1995;9: 427-434. 
32 Lathey JL, Tsou J, Brinker K, et al: Lack of autologous neutralizing antibody to human immunodeficiency virus type 1 (HIV-1) and macrophage tropism are associated with mother-to-infant transmission. J Infect Dis 1999;180:344-350.

- 33 Semba RD, Kumwenda N, Hoover DR, et al: Human immunodeficiency virus load in breast milk, mastitis, and mother-to-child transmission of human immunodeficiency virus type 1. J Infect Dis 1999;180:93-98.

-34 John GC, Nduati RW, Mbori-Ngacha DA, et al: Correlates of mother-to-child human immunodeficiency virus type 1 (HIV-1) transmission: association with maternal plasma HIV-1 RNA load, genital HIV-1 DNA shedding, and breast infections. J Infect Dis 2001; 183:206-212.

35 Miller N: HIV and breastfeeding: what we do and do not know in 2005. Midwifery Today Int Midwife 2006 Spring;(77):38-39.

- 36 Miotti PG, Taha TE, Kumwenda NI, et al: HIV transmission through breastfeeding: a study in Malawi. JAMA 1999;282:744-749.

- 37 Olayinka B, Oni AO, Mbajiorgu FE: Impact of infant feeding practices on the risk of mother to child transmission of HIV-1 in Zimbabwe. J Paediatr Child Health 2000;36: 313-317.

38 Van de Perre P, Simonon A, Msellati P, et al: Postnatal transmission of human immunodeficiency virus type 1 from mother to infant. A prospective cohort study in Kigali, Rwanda. N Engl J Med 1991;325:593-598.

-39 Van de Perre P, Hitimana DG, Simonon A, et al: Postnatal transmission of HIV-1 associated with breast abscess. Lancet 1992;339: 1490-1491.

-40 Nduati R, John G, Mbori-Ngacha D, et al: Effect of breastfeeding and formula feeding on transmission of HIV-1: a randomized clinical trial. JAMA 2000;283:1167-1174.

41 Coutsoudis A, Pillay K, Spooner E, et al: Influence of infant-feeding patterns on early mother-to-child transmission of HIV-1 in Durban, South Africa: a prospective cohort study. South African Vitamin A Study Group. Lancet 1999;354:471-476.

42 Coutsoudis A, Pillay K, Kuhn L, et al: Method of feeding and transmission of HIV-1 from mothers to children by 15 months of age: prospective cohort study from Durban, South Africa. AIDS 2001;15:379-387.

43 Downs JH, Cooper PA: HIV and lactation. Ann Nestlé [Engl] 2007;65:29-38.

-44 Papathakis PC, Rollins NC, Brown KH, et al: Comparison of isotope dilution with bioimpedance spectroscopy and anthropometry for assessment of body composition in asymptomatic HIV-infected and HIV-uninfected breastfeeding mothers. Am J Clin Nutr 2005;82:538-546.

-45 Nduati R, Richardson BA, John G, et al: Effect of breastfeeding on mortality among HIV-1 infected women: a randomised trial. Lancet 2001;357:1651-1655.
46 Newell M: Does breastfeeding really affect mortality among HIV-1 infected women? Lancet 2001;357:1634-1635.

- 47 Coutsoudis A, Coovadia H, Pillay K, Kuhn L: Are HIV-infected women who breastfeed at increased risk of mortality? AIDS 2001;15: 653-655.

48 Sedgh G, Spiegelman D, Larsen U, et al: Breastfeeding and maternal HIV-1 disease progression and mortality. AIDS 2004;18: 1043-1049.

49 Kuhn L, Kasonde P, Sinkala M, et al: Prolonged breast-feeding and mortality up to two years post-partum among HIV-positive women in Zambia. AIDS 2005; 19:16771681.

50 Taha TE, Kumwenda NI, Hoover DR, et al: The impact of breastfeeding on the health of HIV-positive mothers and their children in sub-Saharan Africa. Bull World Health Organ 2006;84:546-554.

51 Breastfeeding and HIV International Transmission Study Group: Mortality among HIV-1-infected women according to children's feeding modality: an individual patient data meta-analysis. J Acquir Immune Defic Syndr 2005;39:430-438.

52 Allard JP, Aghdassi E, Chau J, et al: Effects of vitamin $\mathrm{E}$ and $\mathrm{C}$ supplementation on oxidative stress and viral load in HIV-infected subjects. AIDS 1998;12:1653-1659.

53 Jiamton S, Pepin J, Suttent R, et al: A randomized trial of the impact of multiple micronutrient supplementation on mortality among HIV-infected individuals living in Bangkok. AIDS 2003;17:2461-2469.

54 Fawzi WW, Msamanga GI, Spiegelman D, et al: A randomized trial of multivitamin supplements and HIV disease progression and mortality. N Engl J Med 2004;351:23-32.

55 Fawzi W, Msamanga G, Spiegelman D, Hunter DJ: Studies of vitamins and minerals and HIV transmission and disease progression. J Nutr 2005;135:938-944.

56 Dorosko SM: Vitamin A, mastitis, and mother-to-child transmission of HIV-1 through breast-feeding: current information and gaps in knowledge. Nutr Rev 2005;63:332346.

57 Farias EF, Ong DE, Ghyselinck NB, et al: Cellular retinol-binding protein I, a regulator of breast epithelial retinoic acid receptor activity, cell differentiation, and tumorigenicity. J Natl Cancer Inst 2005;97:21-29.

-58 Seewaldt VL, Caldwell LE, Johnson BS, et al: Inhibition of retinoic acid receptor function in normal human mammary epithelial cells results in increased cellular proliferation and inhibits the formation of a polarized epithelium in vitro. Exp Cell Res 1997;236:1628.

59 Dreyfuss ML, Fawzi WW: Micronutrients and vertical transmission of HIV-1. Am J Clin Nutr 2002;75:959-970.
60 Buck J, Ritter G, Dannecker L, et al: Retinol is essential for growth of activated human B cells. J Exp Med 1990;171:1613-1624.

61 Garbe A, Buck J, Hammerling U: Retinoids are important cofactors in $\mathrm{T}$ cell activation. J Exp Med 1992;176:109-117.

62 Kuhn L, Coutsoudis A, Moodley D, et al: Thelper cell responses to HIV envelope peptides in cord blood: protection against intrapartum and breast-feeding transmission. AIDS 2001;15:1-9.

63 Emmett PM, Rogers IS: Properties of human milk and their relationship with maternal nutrition. Early Hum Dev 1997;49(suppl): S7-S28.

64 Nduati RW, John GC, Richardson BA, et al: Human immunodeficiency virus type 1-infected cells in breast milk: association with immunosuppression and vitamin A deficiency. J Infect Dis 1995;172:1461-1468.

65 Semba RD, Miotti PG, Chiphangwi JD, et al: Maternal vitamin A deficiency and motherto-child transmission of HIV-1. Lancet 1994; 343:1593-1597.

66 Baeten JM, Richardson BA, Bankson DD, et al: Use of serum retinol-binding protein for prediction of vitamin A deficiency: effects of HIV-1 infection, protein malnutrition, and the acute phase response. Am J Clin Nutr 2004;79:218-225.

67 Filteau SM, Morris SS, Abbott RA, et al: Influence of morbidity on serum retinol of children in a community-based study in northern Ghana. Am J Clin Nutr 1993;58: 192-197.

68 Coutsoudis A, Pillay K, Spooner E, et al: Randomized trial testing the effect of vitamin A supplementation on pregnancy outcomes and early mother-to-child HIV-1 transmission in Durban, South Africa. South African Vitamin A Study Group. AIDS 1999; 13:1517-1524.

69 Pillay K, Coutsoudis A, York D, et al: Cellfree virus in breast milk of HIV-1-seropositive women. J Acquir Immune Defic Syndr 2000;24:330-336.

70 Kumwenda N, Miotti PG, Taha TE, et al: Antenatal vitamin A supplementation increases birth weight and decreases anemia among infants born to human immunodeficiency virus-infected women in Malawi. Clin Infect Dis 2002;35:618-624.

71 Iliff PJ, Piwoz EG, Tavengwa NV, et al: Early exclusive breastfeeding reduces the risk of postnatal HIV-1 transmission and increases HIV-free survival. AIDS 2005;19:699-708.

72 Fawzi WW, Msamanga G, Hunter D, et al: Randomized trial of vitamin supplements in relation to vertical transmission of HIV-1 in Tanzania. J Acquir Immune Defic Syndr 2000;23:246-254.

73 Fawzi WW, Msamanga GI, Spiegelman D, et al: Randomised trial of effects of vitamin supplements on pregnancy outcomes and $\mathrm{T}$ cell counts in HIV-1-infected women in Tanzania. Lancet 1998;351:1477-1482. 
-74 Fawzi WW, Msamanga GI, Hunter D, et al: Randomized trial of vitamin supplements in relation to transmission of HIV-1 through breastfeeding and early child mortality. AIDS 2002;16:1935-1944.

75 Villamor E, Saathoff E, Manji K, et al: Vitamin supplements, socioeconomic status, and morbidity events as predictors of wasting in HIV-infected women from Tanzania. Am J Clin Nutr 2005;82:857-865.

76 Coodley GO, Nelson HD, Loveless MO, Folk C: Beta-carotene in HIV infection. J Acquir Immune Defic Syndr 1993;6:272-276.

$\checkmark 77$ Nimmagadda AP, Burri BJ, Neidlinger T, et al: Effect of oral beta-carotene supplementation on plasma human immunodeficiency virus (HIV) RNA levels and CD4+ cell counts in HIV-infected patients. Clin Infect Dis 1998;27:1311-1313.
78 Austin J, Singhal N, Voigt R, et al: A community randomized controlled clinical trial of mixed carotenoids and micronutrient supplementation of patients with acquired immunodeficiency syndrome. Eur J Clin Nutr 2006;60:1266-1276.

79 Semba RD, Lyles CM, Margolick JB, et al: Vitamin A supplementation and human immunodeficiency virus load in injection drug users. J Infect Dis 1998;177:611-616.

80 MacDonald KS, Malonza I, Chen DK, et al: Vitamin A and risk of HIV-1 seroconversion among Kenyan men with genital ulcers. AIDS 2001;15:635-639.
81 Tang AM, Graham NM, Kirby AJ, et al: Dietary micronutrient intake and risk of progression to acquired immunodeficiency syndrome (AIDS) in human immunodeficiency virus type 1 (HIV-1)-infected homosexual men. Am J Epidemiol 1993;138:937-951.

82 Tang AM, Graham NM, Saah AJ: Effects of micronutrient intake on survival in human immunodeficiency virus type 1 infection. Am J Epidemiol 1996;143:1244-1256.

83 Becquet R, Leroy V, Ekouevi DK, et al: Complementary feeding adequacy in relation to nutritional status among early weaned breastfed children who are born to HIV-infected mothers: ANRS 1201/1202 Ditrame Plus, Abidjan, Cote d'Ivoire. Pediatrics 2006; 117:e701-e710 